\title{
Application of Quasi-Classical Approximation to Describe Black Holes
}

\author{
Vasiliy K. Balkhanov \\ Institute of Physical Materials Science of the Siberian Branch \\ of the Russian Academy of Sciences, Ulan-Ude City, Russia \\ Email: ballar@yandex.ru
}

\begin{abstract}
The article found that quasi-classic quantum approach allows to calculate temperature and entropy of black holes. Installed border applicability of quasi classical approximation: the mass of the black hole must be greater than the Planck mass.
\end{abstract}

Key words: Entropy, temperature, quasi-classics

\section{Introduction}

Physics of black holes (BH) is described in many books and reviews [1-8]. It was found that $\mathrm{BH}$ with the classical point of view to the distant observer represents the sky blacker black circle of radius $R=2 \gamma M / c^{2}$. Here $M$ is observed from a distance the mass BH, $\gamma$ - Newton's gravitational constant, $c$ - the speed of light. The availability of mass in BH means that BH has internal energy $E=M c^{2}$. Since the position of a distant observer, in 3D space with nothing selected, the $\mathrm{BH}$ is a ball with the surface area $A=4 \pi R^{2}$. The perfectly smooth surface is called the event horizon. The inclusion of quantum mechanics to two fundamental constant $\gamma$ and $c$ makes even Planck constant options $\hbar$. The emergence $\hbar$ of substantially changes the physics BH. It is determined that the event horizon is no longer perfectly smooth. The presence of a strong gravitational field nearby leads to quantum fluctuations. Space near the event horizon, it boils is heat or stove, of which external space by virtue of quantum tunneling constantly happen with radiation S. Hawking. Such radiation means that BH has temperature and entropy. All these features can be installed from the dimensional considerations, if three permanents $\hbar, c$ and $\hbar$ (from Planck units which are) add a mass $M$ of $\mathrm{BH}$.

The paper suggests a theoretical approach to calculate the characteristics BH. Our approach is based on the fact that the presence of large masses at BH allows you to apply quantum quasi-lassic uncertainty principle as set out in [9]. The attraction of this principle lets you find the condition of its applicability. As further establish a lot of $\mathrm{BH}$ should be considerably more weight.

\section{Quasi-classical Approximation and Tunneling.}

Proceed to the application of quasi-classical principle of uncertainty. We introduce the radial coordinate, $z$, which is taken as a result of quantum fluctuations of the event horizon from his classical equilibrium value. As a result, these fluctuations of the event horizon are always in motion, i.e. have speed $\dot{z}$, and together with it is the momentum $M \dot{z}$. Since the thermal atmosphere boils, she has a scattering of energy that determines the temperature $T$ (energy units) the heat of the atmosphere. According to the quasi-classical principle of uncertainty entered values are subject to the following expression:

$$
T z \sim \dot{\hbar} \dot{z}
$$

Using this ratio, one can find the entropy, $S$, which is stocked in BH. Due to that fluctuations of the event horizon are shifting to a distance of the order of the size of the event horizon, then part of the BH can quantum tunneling evaporate into outer space. The probability of such an event, according to the quasi-classical tunneling, will be proportional to the exhibitor 


$$
\exp \left(-\frac{2}{\hbar} \int_{0}^{R} M \dot{z} d z\right)
$$

Taking the logarithm, we find the entropy $S=-\ln$ (probability), which is blown in the outer space and which, accordingly, has itself $\mathrm{BH}$ :

$$
S=\frac{2}{\hbar} \int_{0}^{R} M \dot{m} d z
$$

Replacing with the formula (1) speed $\dot{z}$ on the $z$ coordinate and integrate, we find:

$$
S=a \frac{M T}{\hbar^{2}} R^{2}
$$

Changing $R$ on $2 \gamma M / c^{2}$, we get:

$$
S=4 a \frac{\gamma^{2}}{\hbar^{2} c^{2}} T M^{3}
$$

Here you have entered numerical multiplier a, which arose due to the mark of proportionality in the formula (1) and the approximate upper limit of integration in the formula (2).

\section{$3 \quad$ Entropy and Temperature.}

Next, you must use the known thermodynamic formulas, set out, for example, [10]. It is known that if the system has entropy $S$ and the energy $E$, then its temperature $T$ can be found from the relationship:

Substituting $E=M c^{2}$, we get:

$$
\frac{1}{T}=\frac{d S}{d E}
$$

$$
\frac{1}{T}=\frac{d S}{c^{2} d M}
$$

Expressing equation (3) temperature $T$ via the entropy $S$, we get a differential equation:

$$
4 a \frac{\gamma^{2}}{\hbar^{2} c^{4}} \frac{M}{S}=\frac{d S}{c^{2} d M}
$$

By integrating it with the initial condition $S(M=0)=0$, we get:

$$
S=\sqrt{2 a} \frac{\gamma}{\hbar c} M^{2}
$$

If you replace a mass $\mathrm{M} \mathrm{BH}$ at radius $\mathrm{R} \mathrm{BH}$ and enter area event horizon, then you get a more familiar expression for entropy $\mathrm{BH}$ :

$$
S=\frac{\sqrt{2 a}}{16 \pi} \frac{C^{3}}{\hbar \gamma} A
$$

Since the Planck units, according to S. Hawking,

$$
S=\frac{1}{4} A, \text { then } \sqrt{2 a}=4 \pi .
$$

Substituting equation (4) into equation (3), we find the famous phrase for the temperature of BH:

$$
T=\frac{1}{8 \pi} \frac{\hbar c^{3}}{\gamma} \frac{1}{M}
$$

\section{Border Applicability of Quasi-classical Approximation.}

You can now consider the applicability of the quasi-classical approximation. Its applicability, as it is known, means that the exponential multiplier in equation (2) must be significantly more than 1 . This constraint leads to specifying that $S>>1$, or, as can be seen from equation (4): 


$$
M>\sqrt{\frac{\hbar c}{\gamma}}=\text { Planck mass. }
$$

Thus, the classical approximation is applicable for BH, massing significantly more Planck mass.

\section{$5 \quad$ Holographic Principle}

The holographic principle of the internal structure of the $\mathrm{BH}$ fully reflected its event horizon. The principle means that the entropy of the $\mathrm{BH}$ should be equal to the entropy fluctuations of event horizon, i.e. should be given by the expression (4). Together with equity entropy will be equal and low heat of the atmosphere and temperature of BH consistent with thermodynamic equilibrium. Since the entropy is proportional to the square of one side event horizon, and, on the other hand, is proportional to the square of the mass of $\mathrm{BH}$, the Planck units with accuracy to numerical multipliers will be equal $M^{2}=$ $A$. Or, taking into account all fundamental constants, the holographic principle mathematically means that

$$
\frac{\gamma}{\hbar c} M^{2}=\frac{c^{3}}{\hbar \gamma} A
$$

Ratio reduced to linear connection, the size of the event horizon and masses of BH. Received 100 years ago Schwarzschild solution already contained the holographic principle!

\section{Conclusion.}

Quasi-classical approximation currently applies to all $\mathrm{BH}$ in the universe, and allows you to get all of the known characteristics of the $\mathrm{BH}$.

Acknowledgments. This article is prepared for the budget draft of Laboratory Electromagnetic Diagnostics Institute of Physical Materials Science of the Siberian Branch RAS.

\section{References}

1. A.M. Cherepashchuk, Phys. Usp. 57, 359-376 (2014); DOI: 10.3367/UFNr.0184.201404d.0387.

2. V.P. Frolov, I.D. Novikov, Black Hole Physics: Basic Concepts and New Developments, Dordrecht, Boston, London: Kluwer Academic Publishers, 1998.

3. Barton Cvibax, A First Course in String Theory, Second edition, Massachusetts Institute of Technology, 2009.

4. S. Chandrasekhar, The Mathematical Theory of Black Holes, Chicago, New York: Oxford University Press, 1983.

5. I.D. Novikov, Phys. Usp. 59, 713-715 (2016); DOI: 10.3367/UFNe.2015.12.037768.

6. N.D. Birrek, P.C.W. Davies Quantum Fields in Curved Space, London, New York, New Rochelle, Melbourne, Sydney: Cambridge University Press, 1982.

7. Leonard Susskind, The Black Hole War: My Battle with Stephen Hawking to Make the World Safe for Quantum Mechanics, Little, Brown and Company, 2008.

8. M. Rees, R. Ruffini, J.A. Wheeler, Black Holes, Gravitional Waves and Cosmology: An Introduction to Current Research, New York, London, Paris: Gordon and Breach Science Publishers, 1974.

9. L. D. Landau and E. M. Lifshitz, Quonturn Mechunics: Non-relutivistic Theory, 3rd Ed., Oxford: Pergamon Press, 1977. §16.

10.L.D. Landau, E. M. Lifshits, Statistical Physics, Pt. 1, 3rd Ed., Oxford: Pergamon Press, 1980. 